\title{
HDAC inhibitors, trichostatin A and valproic acid, increase E-cadherin and vimentin expression but inhibit migration and invasion of cholangiocarcinoma cells
}

\author{
JOON HO WANG $^{1}$, EUN JEOUNG LEE ${ }^{2}$, MEIYING $\mathrm{JI}^{2}$ and SEON MEE PARK ${ }^{2,3}$ \\ ${ }^{1}$ Department of Internal Medicine, Konkuk University Chungju Hospital, Chungju, Chungcheongbuk 27478; \\ ${ }^{2}$ Department of Internal Medicine, Chungbuk National University College of Medicine, Cheongju; \\ ${ }^{3}$ Department of Internal Medicine, Chungbuk National University Hospital, Cheongju, \\ Chungcheongbuk 28644, Republic of Korea
}

Received November 12, 2017; Accepted May 10, 2018

DOI: 10.3892/or.2018.6441

\begin{abstract}
The effects of histone deacetylase (HDAC) inhibitors on epithelial-mesenchymal transition (EMT) differ in various cancers. E-cadherin is a cell-to-cell adhesion protein, whereas accumulation of vimentin is related to the development of the spindle shape of the mesenchymal cell phenotype. We investigated the EMT phenotypes of human cholangiocellular carcinoma HuCC-T1, JCK and SNU-1079 cell lines. To this end, we measured the expression of E-cadherin or zonula occludens (ZO)-1 and vimentin, epithelial and mesenchymal cell markers, respectively, using real-time reverse transcription-polymerase chain reaction, western blotting, and immunofluorescence microscopy following treatment with trichostatin A (TSA, $200 \mathrm{nM}$ ) or valproic acid (VPA, $0.5 \mathrm{mM}$ ) with or without gemcitabine (GEM, $50 \mathrm{nM}$ ) for $24 \mathrm{~h}$. In addition, we performed cell morphology, migration, and invasion assays. HuCC-T1 cells changed from spindle- to rectangular-shaped after co-treatment with GEM and TSA or VPA. Furthermore, cells co-treated with GEM and TSA or VPA exhibited protein levels of E-cadherin or ZO-1 that were higher than those in cells treated with GEM alone, indicating stronger inhibition of EMT. However, vimentin expression was also increased. Confocal microscopy revealed enhanced expression of E-cadherin or ZO-1 and vimentin in all three cell lines. Migration and invasion were inhibited in HuCC-T1 cells co-treated with
\end{abstract}

Correspondence to: Professor Seon Mee Park, Department of Internal Medicine, Chungbuk National University College of Medicine, 1 Chungdae-ro, Seowon-gu, Cheongju, Chungcheongbuk 28644, Republic of Korea

E-mail: smpark@chungbuk.ac.kr

Abbreviations: CCA, cholangiocellular carcinoma; EMT, epithelial-mesenchymal transition; GEM, gemcitabine; HDAC, histone deacetylase inhibitor; TSA, trichostatin A; VPA, valproic acid

Key words: cell invasion, cell migration, cholangiocarcinoma cells, epithelial-mesenchymal transition, gemcitabine, histone deacetylase inhibitor
GEM and TSA or VPA, compared to those treated with GEM alone. In conclusion, co-treatment of cholangiocarcinoma cells with TSA or VPA and GEM suppressed EMT with tolerable cytotoxicity. However, the HDAC inhibitors augmented both E-cadherin and vimentin expression and their effects varied in different cholangiocarcinoma cell lines. Therefore, the clinical use of HDAC inhibitors in biliary cancer should be considered cautiously.

\section{Introduction}

Cholangiocellular carcinoma (CCA) is more prevalent in Asian countries than it is in Western countries. However, the incidence of intrahepatic CCA has increased worldwide. Most patients present with advanced disease, and less than $30 \%$ are eligible for curative resection. After surgery, the 5-year survival rate is $20 \%$, and chemotherapy and radiation have not yet been proven to prolong survival time (1). Chronic inflammation, cytokines and molecular alterations induce genetic and epigenetic changes, which contribute to the initiation, promotion and progression of CCA (2). Acetylation or deacetylation of the lysine residue in histones modifies chromatin through the competitive action of histone acetyltransferases (HATs) and histone deacetylases (HDACs). HDACs promote carcinogenesis by repressing tumor-suppressor genes, and they are overexpressed in numerous types of tumors (3). HDACs are divided into four classes based on their homology to yeast HDACs.

HDAC inhibitors are also divided into four classes based on their chemical structures: Hydroxamates [including trichostatin A (TSA) and suberoylanilide hydroxamic acid (SAHA)], aliphatic acids [including valproic acid (VPA)], cyclic peptides, and benzamides (4). Hyperacetylation by HDAC inhibitors is associated with chromatin decondensation, leading to the transcription of genes with anticancer effects such as antiproliferation, cell cycle arrest, differentiation and apoptosis. TSA exhibits antiproliferative effects on biliary tract cancer cell lines (5). SAHA and VPA act synergistically with the chemotherapeutic drug 5-fluorouracil (5-FU) against CCA cells (6). VPA augments the effect of 5-FU on CCA and pancreatic cancer cells, possibly by increasing apoptosis or 
p21 expression (7). These results suggest that HDAC inhibitors have therapeutic potential for cancers of the biliary tract, and act synergistically with conventional chemotherapeutic drugs.

Malignant cells undergo epithelial-to-mesenchymal transition (EMT), a process by which epithelial cells lose their cell-cell adhesion properties, acquire a non-polarized mesenchymal phenotype, and express proteins such as vimentin, Snail, zinc finger E-box-binding homeobox 1 (Zeb1) and Twist family bHLH transcription factor 1 (Twist) (8). The expression of vimentin and loss of E-cadherin are associated with carcinogenesis and are linked to tumor cell migration and invasion (9). Histone modification has been shown to play a key role in controlling EMT. Specifically, recent reports have shown that HDAC inhibitors are associated with suppression of EMT in various solid tumors (10-14). Numerous studies have reported that various HDAC inhibitors reverse or attenuate EMT through the upregulation of E-cadherin in different solid tumors such as hepatocellular carcinoma (HCC) (10), breast $(11,12)$, esophageal (13), and ovarian (14) cancers.

In addition, HDAC inhibitors attenuate EMT induced by transforming growth factor (TGF)- $\beta 1$ in different cells, such as hepatocytes (15), retinal pigment epithelial (16), lens epithelial (17), and renal epithelial cells (18). These results suggest that HDAC inhibitors may be applied therapeutically to reduce EMT. However, recent studies have also reported that HDAC inhibitors may induce EMT and stem cell properties, as well as enhance metastasis and invasion in prostate (19), head and neck squamous cell carcinoma (20), nasopharyngeal carcinoma (21), and colorectal (22) cancer. In contrast, Sakamoto et al (23) reported that the HDAC inhibitor vorinostat suppressed TGF- $\beta$-induced EMT in biliary tract cancer. The effect of HDAC inhibitors on the suppression of EMT appears to differ according to the cell type examined, the specific HDAC inhibitor used, and its dose. Whether HDAC inhibitors inhibit or induce EMT in cholangiocarcinoma needs to be clarified.

Therefore, in this study, we investigated the effect of the HDAC inhibitors TSA and VPA on the EMT of CCA cells alone and in combination with the chemotherapeutic drug gemcitabine (GEM). We evaluated the expression of E-cadherin or zonula occludens (ZO-1), epithelial markers, and vimentin, a mesenchymal marker. EMT was evaluated by observing cell morphology and performing migration and invasion assays.

\section{Materials and methods}

Cell lines and culture condition. We used three CCA cell lines. HuCC-T1 and SNU-1079 cells were purchased from the American Type Culture Collection (ATCC, Rockville, MD, USA) and the Korea Cell Line Bank (Seoul, Korea), respectively, and JCK cells were a kind gift from D.H. Kim, from Cheonbuk National University. The cell lines were maintained in Dulbecco's modified Eagle's medium (DMEM; Sigma-Aldrich, St. Louis, MO, USA) containing 10\% heat-inactivated fetal bovine serum (FBS; Sigma-Aldrich), $100 \mathrm{U} / \mathrm{ml}$ potassium penicillin (Sigma-Aldrich), $100 \mathrm{~g} / \mathrm{ml}$ streptomycin, $2 \mathrm{mM}$ glutamine (Sigma-Aldrich) and $20 \mathrm{mM}$ sodium bicarbonate (Sigma-Aldrich). The cells were incubated with $5 \% \mathrm{CO}_{2}$ in $95 \%$ humidity in a $37^{\circ} \mathrm{C}$ chamber. The growth medium was changed every 3 days.
Real-time quantitative polymerase chain reaction ( $R T$ - $q P C R)$. Total RNA was isolated using the RNase Mini kit (Qiagen, Inc., Valencia, CA, USA). Residual DNA was removed using an RNase free DNase kit (Qiagen, Inc.). Applied Biosystems ${ }^{\mathrm{TM}}$ High-Capacity RNA-to-cDNA kit (Thermo Fisher Scientific, Inc., Waltham, MA, USA) was used to reverse-transcribe $1 \mu \mathrm{g}$ RNA into cDNA, according to the manufacturer's instructions. RT-qPCR was performed using specific primers to quantify gene expression using Applied Biosystems ${ }^{\mathrm{TM}}$ SYBR Green RT-PCR reagents (Thermo Fisher Scientific, Inc.).

The relative amount of mRNA was normalized to the expression of actin. The primer sequences used in this study were as follows: Actin: Forward, 5'-GTTGTCGACGACGAG CG-3' and reverse, 5'-GCACAGAGCCTCGCCTT-3'; zonula occludens (ZO-1): Forward, 5'-CCCCACTCTGAAAATGAG GA-3' and reverse, 5'-GGGAACAACATACAGTGACGC-3; E-cadherin: Forward, 5'-TTCTGCTGCTCTTGCTGTTT-3' and reverse, 5'-TGGCTCAAGTCAAAGTCCTG-3; and vimentin: Forward, 5'-GCCCTTAAAGGAACCAATGA-3' and reverse, 5'-AGCTTCAACGGCAAAGTTCT-3'. The PCR reactions were repeated three times in three independent experiments. The expression of ZO-1 was determined for SNU-1079 cells.

Western blot analysis. Total protein was extracted, and the protein concentration was measured using the Bradford DC protein assay (Bio-Rad Laboratories, Inc., Hercules, CA, USA). Subsequently, 20-40 $\mu$ g protein from each sample was separated using 12\% Bis-Tris polyacrylamide gel electrophoresis and blotted onto Immobilon transfer membranes (EMD Millipore, Billerica, MA, USA). The blots were immunostained with E-cadherin (1:100; cat. no. 701134; Invitrogen; Thermo Fisher Scientific), ZO-1 (1:200; cat. no. 61-7300; Zymed Laboratories ${ }^{\circledR}$; Thermo Fisher Scientific) and vimentin (1:200; cat. no. V2258; Sigma-Aldrich; Merck KGaA, Darmstadt, Germany) primary antibodies at $4^{\circ} \mathrm{C}$ overnight, followed by incubation with the secondary antibody at room temperature for $1 \mathrm{~h}$. The expression of ZO-1 was determined for SNU-1079 cells. The proteins were visualized using enhanced chemiluminescence (ECL) with Plus Western Blotting Detection reagents (iNtRon Biotechnology, Daejeon, Korea), followed by exposure to X-ray film (LAS-3000 mini; Fuji Film, Tokyo, Japan).

Immunofluorescence microscopy. For the immunofluorescence microscopy analysis, the CCA cell lines were seeded on individual sterile coverslips placed in the wells of 4-well plates and incubated for $24 \mathrm{~h}$. Subsequently, the cells were treated with $200 \mathrm{nM}$ TSA or $0.5 \mathrm{mM}$ VPA diluted in DMEM with $1 \%$ FBS for $48 \mathrm{~h}$. After washing the cells with PBS, they were fixed in $3.7 \%$ paraformaldehyde for $20 \mathrm{~min}$ at room temperature. The paraformaldehyde was removed, the cells were washed with PBS, incubated with blocking solution for $30 \mathrm{~min}$ to prevent non-specific binding, and then incubated with anti-E-cadherin (1:100; cat. no. 14472; Cell Signaling Technology, Inc., Danvers, MA, USA), anti ZO-1 (1:200; Zymed Laboratories ${ }^{\circledR}$; Thermo Fisher Scientific, Inc.) and anti-vimentin (1:200; cat. no. VIM-572-L-CE; Leica Microsystems, Wetzlar, Germany) primary antibodies overnight at $4^{\circ} \mathrm{C}$ on a rocking platform. The expression of ZO-1 was determined for SNU-1079 cells. The washed slides were incubated for $1 \mathrm{~h}$ at room temperature with 1:100 dilutions of Alexa-488 anti-rabbit $\operatorname{IgG}(\mathrm{H}+\mathrm{L})$ and Alexa-568 goat anti-mouse $\operatorname{IgG}(\mathrm{H}+\mathrm{L})$ 


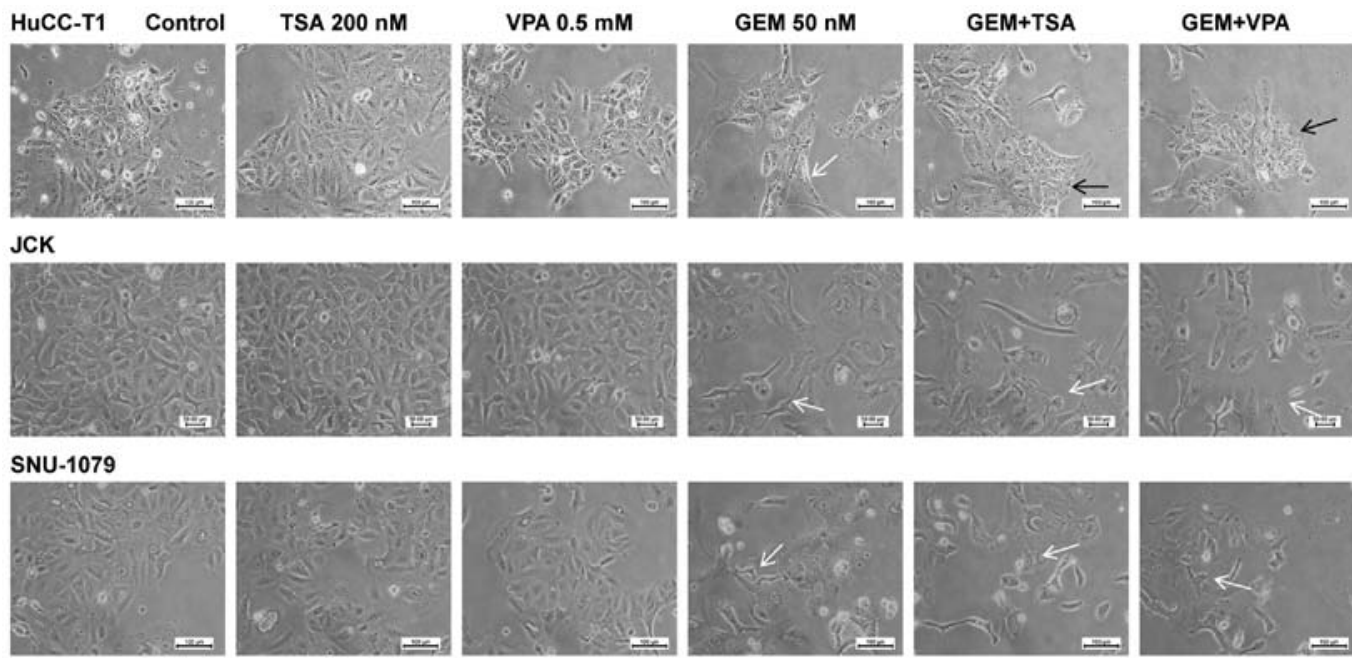

Figure 1. Trichostatin A (TSA) or valproic acid (VPA) attenuate the gemcitabine (GEM)-induced mesenchymal phenotype. Cholangiocarcinoma cells treated with GEM alone for $48 \mathrm{~h}$ exhibited a fibroblastic-type phenotype (white arrows) whereas cells treated with TSA or VPA maintained their original epithelial cell morphology (original magnification, x100). Co-treatment with TSA or VPA and GEM attenuated spindle-like morphological changes (white arrows) in human cholangiocellular carcinoma (HuCC-T1) cells compared to GEM alone, whereas no additive effects of TSA or VPA co-treatment with GEM were observed in JCK and SNU-1079 cells (black arrows).

secondary antibodies (both from Molecular Probes Inc.; Thermo Fisher Scientific). The cells were washed again, mounted with Dako Vectashield mounting medium (Agilent Technologies, Inc., Santa Clara, CA, USA), and examined using a Leica Zeiss optics microscope at the Core Facility of Chungbuk National University.

Cell invasion and migration assays. We performed cell invasion and migration assays using HuCC-T1 cells. Briefly, HuCC-T1 cells were seeded in 96-well plates over a homogeneous, thin layer of fibronectin (BD Biosciences, Bedford, MA, USA) in Millicell cell culture inserts (EMD Millipore, Billerica, MA, USA) containing polycarbonate filter membranes with $8-\mu \mathrm{m}$ diameter pores. The invasion assay was performed using Transwell chambers coated with Matrigel prior to cell seeding. Control group tumor cells were maintained in DMEM supplemented with $1 \%$ FBS and $1 \%$ antibiotics, and the cells in the HDAC inhibitor group were treated with $50 \mathrm{nM}$ GEM, $200 \mathrm{nM}$ TSA, or $0.5 \mathrm{mM}$ VPA diluted in the medium. The lower chamber contained DMEM supplemented with $10 \%$ FBS and $1 \%$ antibiotics. After plating, cells were incubated for $24 \mathrm{~h}$ at $37^{\circ} \mathrm{C}$ in a $5 \%$ $\mathrm{CO}_{2}$-humidified incubator. Invasive cells in the lower chamber were stained with hematoxylin and eosin (H\&E). Images were acquired using a QImaging ExiAqua monochrome digital camera attached to a Nikon Eclipse 80i Microscope (Nikon, Melville, NY, USA) and visualized using the QCapture Pro software (pro 5.1; QImaging Corp., Surrey, BC, Canada). HuCC-T1 cells were allowed to grow in DMEM containing 10\% FBS confluently in a 6-well plate. A central linear wound was made with a 200-ml sterile pipette tip and then the cells were washed twice with PBS. Afterwards, the HDAC inhibitor group was treated with $50 \mathrm{nM}$ GEM, $200 \mathrm{nM}$ TSA, or $0.5 \mathrm{mM}$ VPA diluted in the medium with $1 \%$ FBS. Plates were photographed after 0,24 and $48 \mathrm{~h}$.

Cytotoxicity assay. Cell viability was assessed using the Promega cell proliferation 3-(4,5-dimethylthiazol-2-yl)-5-(3-carboxym ethoxyphenyl)-2-(4-sulfophenyl)-2H-tetrazolium (MTS) assay kit (Promega, Madison, WI, USA). Briefly, HuCC-T1 cells were seeded in 96-well plates at a density of $1 \times 10^{3}$ per well and incubated with increasing concentrations of TSA, VPA, and GEM for $48 \mathrm{~h}$, and concentration-response curves were calculated. Subsequently, dead cells were washed away, and the attached cells were incubated with MTS, followed by cell viability detection using microplate reader Model-680 (Bio-Rad Laboratories). All experiments were performed in triplicate.

We performed a cytotoxicity assay for gemcitabine, TSA, and VPA for the three CCA cell lines. For gemcitabine and TSA we selected concentrations that inhibit cell proliferation by $0-20 \%$. For valproic acid, we selected a tolerable toxicity to humans, $<0.5 \mathrm{mM}$.

Statistical analysis. Data were analyzed with SPSS software version 18.0 (SPSS Inc., Chicago, IL, USA). Continuous variables are presented as means \pm standard deviation and were compared between the groups by one-way analysis of variance (ANOVA) followed by Turkey's multiple comparison tests. Differences with P-values $<0.05$ were considered significant.

\section{Results}

Cell morphology. Morphological changes were most prominent in the HuCC-T1 cells, which were elongated following treatment with GEM alone. Notably, after co-treatment with GEM and TSA or VPA the cells reverted to a rectangular shape. JCK and SNU-1079 cells treated with GEM exhibited a spindle-shaped morphology. However, co-treatment with GEM and TSA or VPA did not show an additive effect (Fig. 1).

mRNA expression of E-cadherin (or ZO-1) and vimentin. In the HuCC-T1 cells, mRNA expression of E-cadherin increased following treatment with TSA, GEM, and co-treatment with GEM and TSA or VPA. In JCK cells, expression of E-cadherin was increased by GEM and co-treatment with GEM and TSA or VPA. In SNU-1079 cells, mRNA levels of 
HuCC-T1

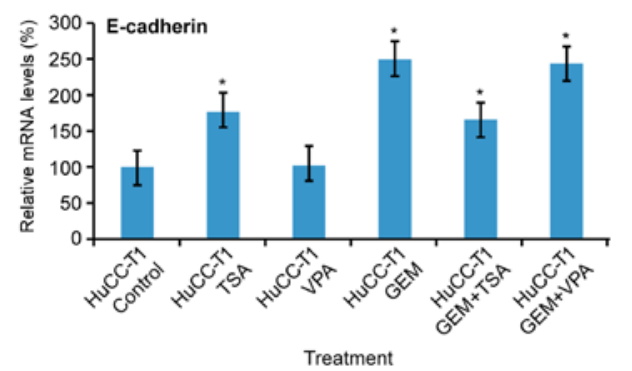

JCK

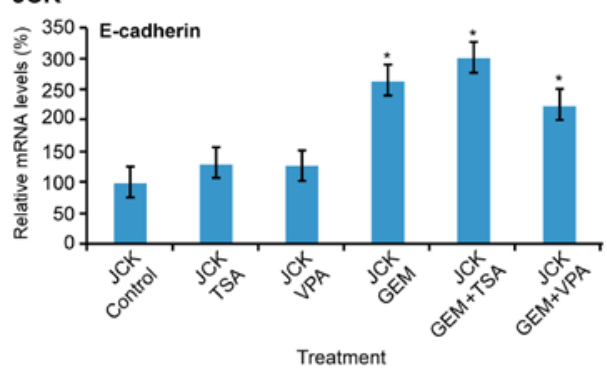

SNU-1079

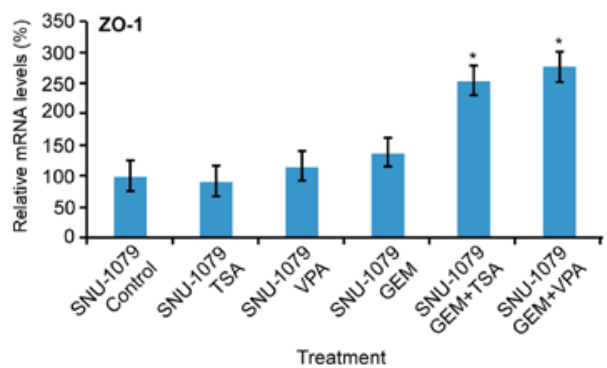

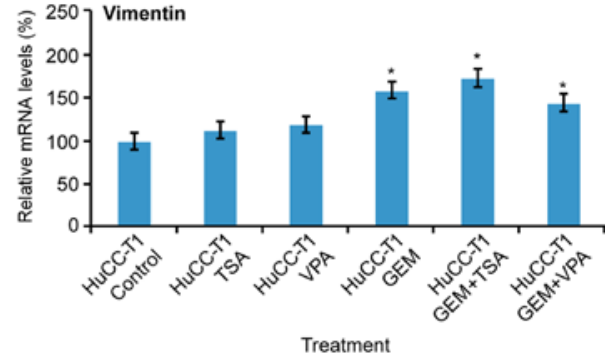
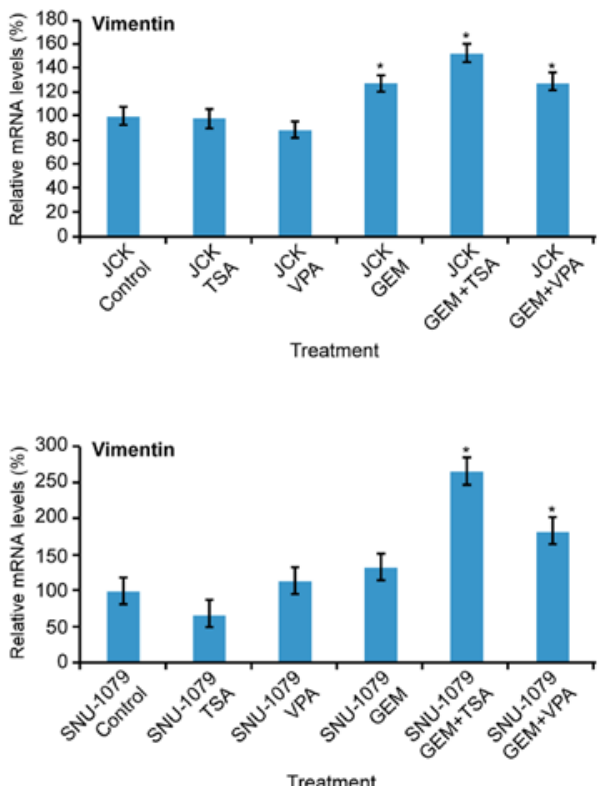

Figure 2. Relative mRNA levels of E-cadherin, zonula occludens (ZO)-1, and vimentin in three cholangiocarcinoma cell lines. Human cholangiocelluar carcinoma HuCC-T1, JCK and SNU-1079 cells treated with trichostatin A (TSA) or valproic acid (VPA) with gemcitabine (GEM) showed higher E-cadherin or ZO-1 and vimentin expression levels than levels noted in the control cells ( $\mathrm{P}<0.05)$. Data are shown as means \pm standard error (SE). Relative expression normalized to the reference gene actin and measured using real-time quantitative reverse transcription-polymerase chain reaction (RT-qPCR).

ZO-1 were increased by co-treatment with GEM and TSA or VPA. Vimentin levels were increased by GEM and TSA or VPA in all three cell lines. Co-treatment with GEM and TSA augmented vimentin expression compared to GEM treatment alone in all three cell lines (Fig. 2).

Protein expression of E-cadherin (or ZO-1) and vimentin. In HuCC-T1 cells, expression of E-cadherin increased in the presence of TSA or GEM, compared to levels in the control cells. Co-treatment of cells with GEM and TSA increased the expression of E-cadherin more than treatment with GEM alone. However, VPA did not induce any change. In JCK cells, treatment with GEM, TSA and VPA increased the expression of E-cadherin compared to that in the control cells. Notably, compared to the cells treated with GEM alone, expression of E-cadherin did not increase when cells were co-treated with GEM and TSA or VPA. In SNU-1079 cells, treatment with GEM, TSA and VPA increased expression of ZO-1 compared to that in the control cells. Furthermore, cells co-treated with GEM and TSA or VPA showed higher expression levels of ZO-1 than cells treated with GEM alone. The expression of vimentin slightly increased in all three cell lines following co-treatment with GEM and TSA or VPA, as well as with GEM alone (Fig. 3). Immunofluorescent staining revealed that

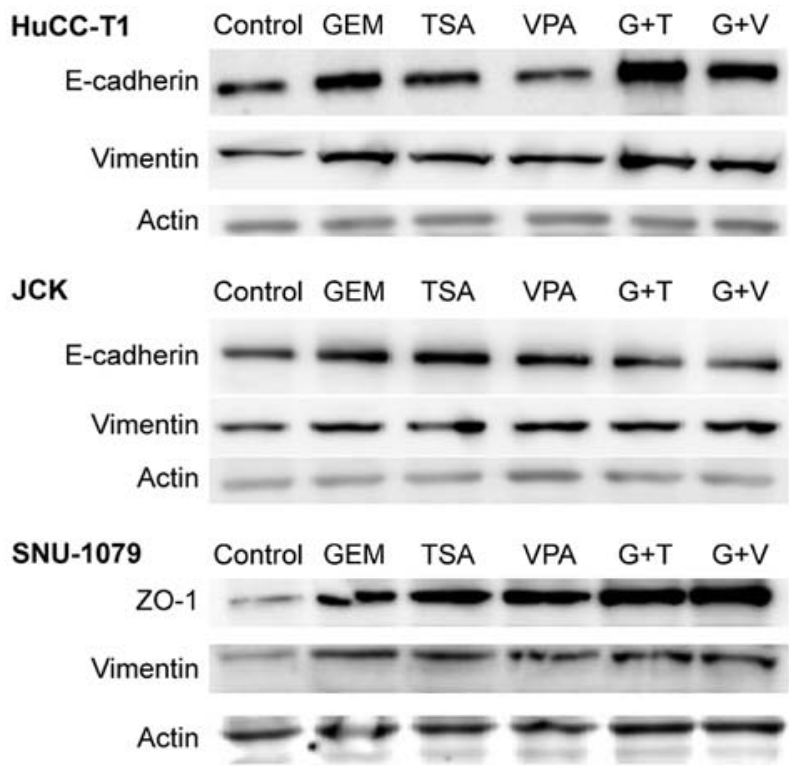

Figure 3. E-cadherin, zonula occludens ( $\mathrm{ZO})-1$, and vimentin protein expression levels in human cholangiocellular carcinoma HuCC-T1, JCK and SNU-1079 cell lines treated with trichostatin A (TSA) or valproic acid (VPA) with or without gemcitabine (GEM) for $24 \mathrm{~h}$. Histone deacetylase (HDAC) inhibitors increased E-cadherin, ZO-1 and vimentin expression in all three cell lines. G+T, GEM and TSA; G+V, GEM and VPA. 
A

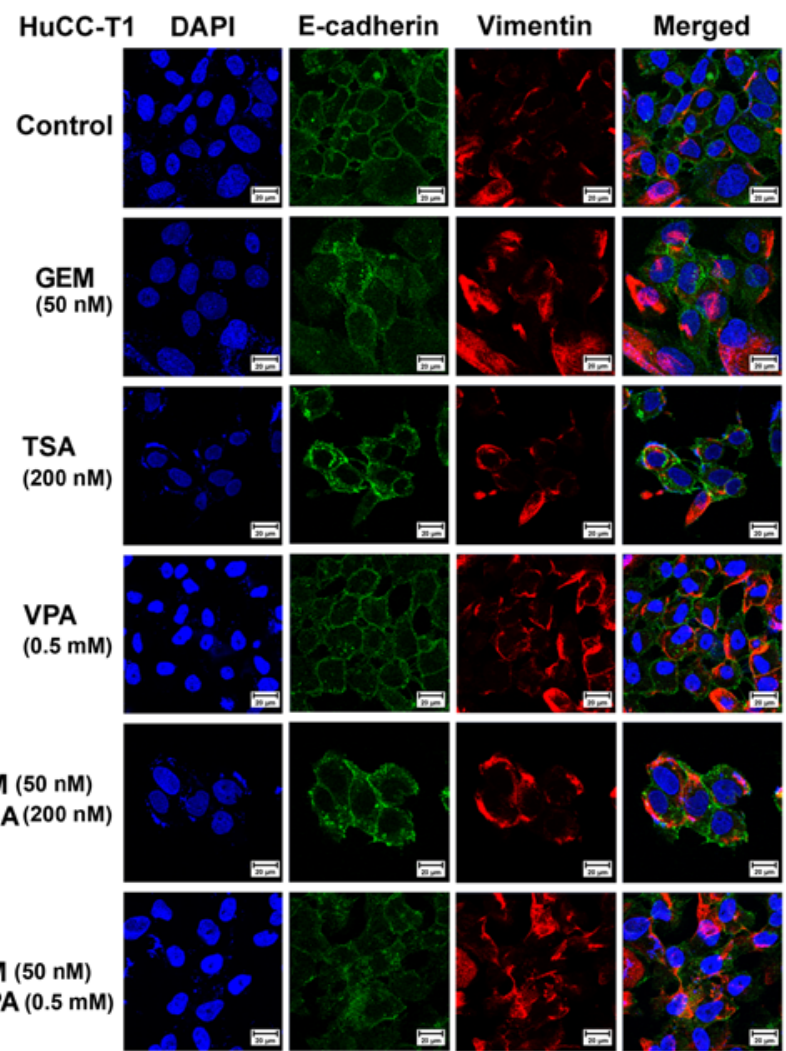

B

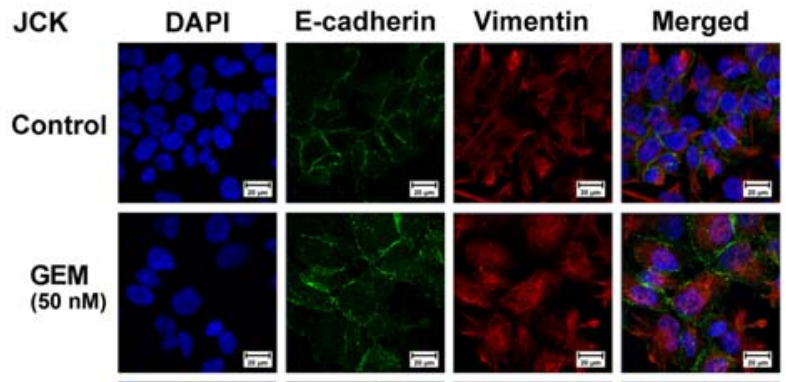

TSA

(200 nM)
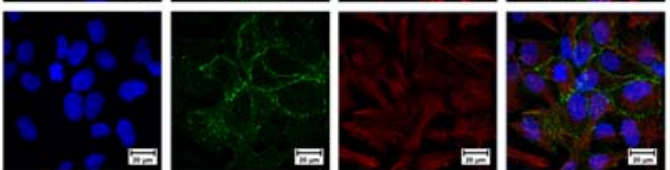

VPA

(0.5 mM)
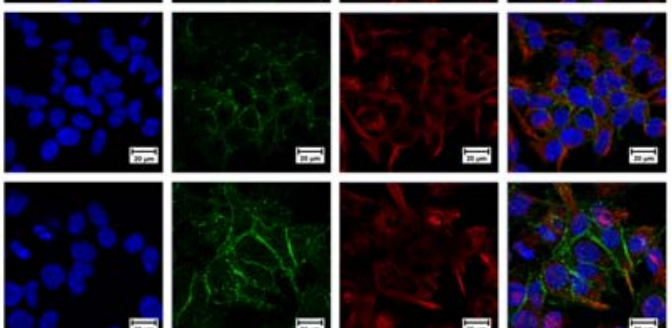

GEM (50 nM)

+ TSA $(200 \mathrm{nM})$
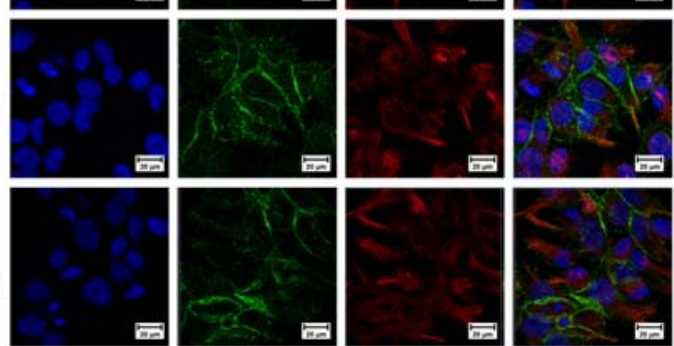

C

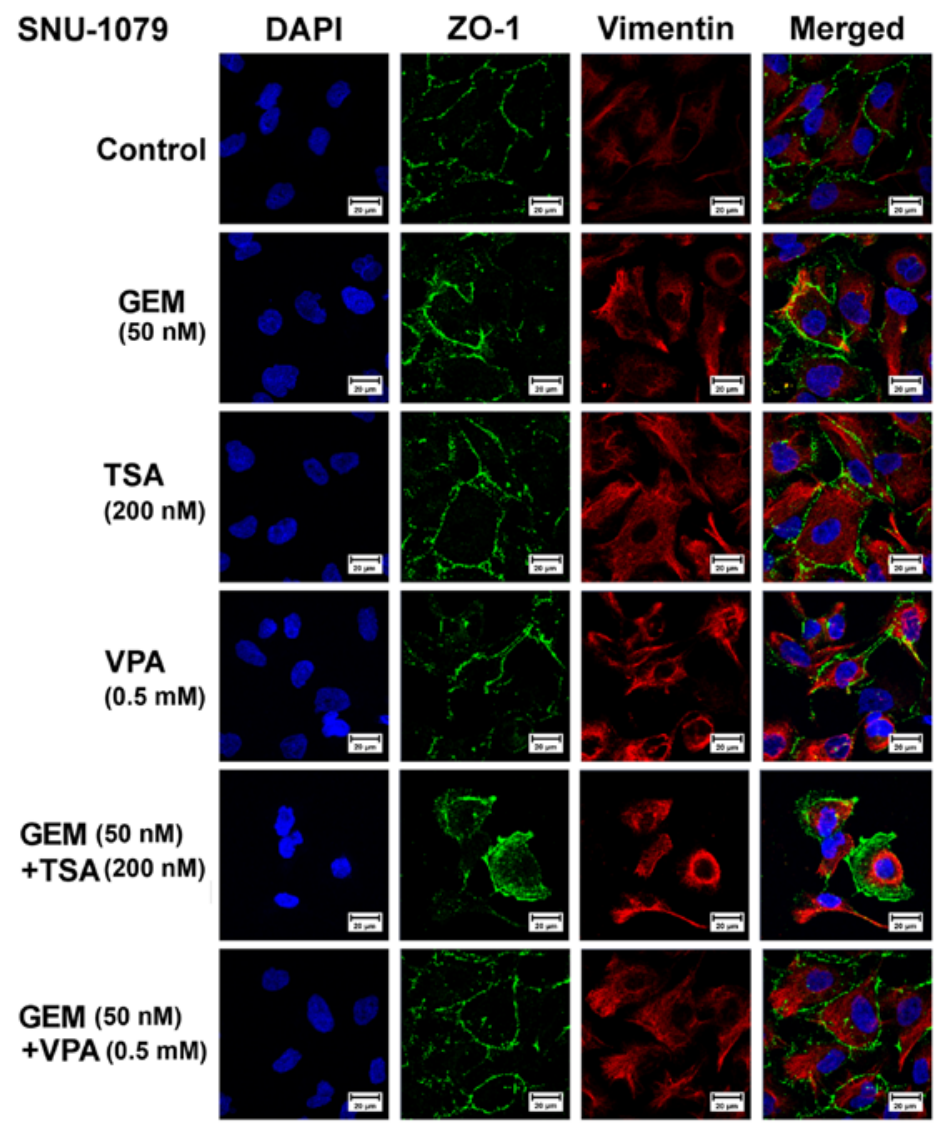

Figure 4. Immunofluorescence microscopy of E-cadherin, zonula occludens (ZO)-1 and vimentin in human cholangiocellular carcinoma (A) HuCC-T1, (B) JCK and (C) SNU-1079 cells. Cholangiocarcinoma cells treated with trichostatin A (TSA, $200 \mathrm{nM}$ ) or valproic acid (VPA, $0.5 \mathrm{mM}$ ) alone or with gemcitabine (GEM, $50 \mathrm{nM}$ ) exhibited increased E-cadherin expression in the HuCC-T1 and JCK cells, ZO-1 in SNU-1079, and vimentin in all cell lines compared to the control cells. E-cadherin or ZO-1 (green), vimentin (red), 4',6-diamidino-2-phenylindole (DAPI, blue), and merged images (original magnification, $\mathrm{x} 400)$. 
E-cadherin or ZO-1 expression in the membranes of HuCC-T1, J-CK, and SNU 1079 cells was increased by treatment with TSA or VPA and GEM compared with GEM treatment alone. However, vimentin expression in the cytoplasm was also increased in all three CCA cell lines (Fig. 4).

Migration and invasion assays. The migration assays after treatment of HuCC-T1 cells with GEM alone showed augmented migration areas, which decreased in cells co-treated with GEM and TSA or VPA. Treatment with TSA or VPA alone showed a decrease or no change in the migration area, respectively (Fig. 5A). The invasion assays showed that cell invasion was decreased by treatment with GEM, TSA, or VPA. GEM and TSA had an additive effect on inhibition of cell invasion, whereas GEM and VPA did not (Fig. 5B).

Cell proliferation. Cell proliferation was assessed in the presence of single or co-treatment with GEM, TSA, and VPA. We investigated the potential dose-related response of cells exposed to increasing concentrations of GEM with or without TSA or VPA. We evaluated the effects of a fixed concentration of GEM $(50 \mathrm{nM})$ and the HDAC inhibitors TSA or VPA (200 $\mathrm{nM}$ or $0.5 \mathrm{mM}$, respectively) on EMT, as these concentrations slightly inhibited cell proliferation (Fig. 6).

\section{Discussion}

This study demonstrated that the HDAC inhibitors, trichostatin A (TSA) and valproic acid (VPA), increased the expression levels of both E-cadherin and vimentin but inhibited the migration and invasion of cholangiocellular carcinoma (CCA) cells, and this effect was enhanced by co-treatment with the chemotherapeutic drug gemcitabine (GEM).

Epithelial-mesenchymal transition (EMT), which confers invasiveness and migratory properties to cells, is the initial step in carcinogenesis and is associated with poor outcome and resistance to chemotherapy (24). EMT comprises modifications of gene expression that permit concurrent epithelial phenotype repression and mesenchymal phenotype activation. E-cadherin and ZO-1 maintain cell-to-cell adhesion, and $\mathrm{N}$-cadherin, vimentin, fibronectin, a-smooth muscle actin, and matrix metalloproteinases constitute intermediate filaments that are detected in mesenchymal cells. EMT-inducing transcription factors (EMT-TFs), such as SNAIL, ZEB, and TWIST, are highly expressed in CCA cells (25). In this study, we selected E-cadherin as an epithelial marker and vimentin as a mesenchymal cell marker. We used ZO-1 as an epithelial marker for SNU-1079 cells, in which E-cadherin is lost owing to hypermethylation (26).

The TGF- $\beta$ signaling pathway plays a major role in EMT and increases the expression of Twist, $\mathrm{N}$-cadherin, and vimentin (27). TSA was found to suppress TGF- $\beta$-induced downregulation of E-cadherin and increase the expression of DNA binding 2 and bone morphogenic protein-7, which in turn inhibited TGF- $\beta$-induced EMT in human renal epithelial cells (18). These findings are consistent with our observation that TSA or VPA upregulated E-cadherin levels in CCA cells. Specifically, we investigated the expression of E-cadherin following exposure of cells to HDAC inhibitors alone or in combination with GEM. Despite some differences between the cell lines, the expression of E-cadherin increased following treatment with the HDAC inhibitors, and this effect was synergistic with GEM.

The human vimentin gene (VIM) includes a TATA box, eight putative GC-boxes, a nuclear factor (NF)- $\mathrm{B}$ binding site, a polyoma enhancer activator 3-binding site, and proximal silencer elements, suggesting that gene regulation of VIM is associated with various EMT-specific and general transcription factors (28-31). For example, zinc-finger binding protein 89 is a negative regulator of vimentin, which recruits HDAC1 at the VIM promoter. Treatment with TSA releases HDAC1 from the promoter and activates VIM expression (32). Vorinostat, an HDAC inhibitor, was found to increase the expression and the acetylation of the EMT-TF Snail and decrease its degradation in nasopharyngeal carcinoma cells. In turn, Snail increased VIM expression (21). TSA suppressed EMT by upregulating E-cadherin and downregulating vimentin, by suppressing Slug expression in colorectal and prostate cancer. Specifically, TSA was found to increase HDAC1 and 2 on the promoter of Slug, decreasing the expression of Slug and consequently that of vimentin (33). Therefore, HDAC inhibitors have been indicated as both inducers and repressors of vimentin. The specific effect probably depends on the cell type and the HDAC inhibitor used.

The present study showed that TSA or VPA increased not only the expression of E-cadherin but also that of vimentin. However, the effects on vimentin expression were less than those on E-cadherin. Therefore, the effect of vimentin has limited action on cell motility and wound healing potential. This would justify the reversion to the epithelial phenotype, despite the elevation of vimentin expression. This study revealed that the invasion and wound healing abilities of HuCC-T1 were cells decreased following co-treatment with one of the HDAC inhibitors in addition to GEM, indicating suppression of EMT. Additionally, HuCC-T1 cell morphology reverted from an elongated to a rectangular shape after co-treatment with TSA or VPA in addition to GEM.

Han et al (34) reported that TSA induced a mesenchymallike morphology and vimentin expression in human gastric and breast cancer cells. However, E-cadherin expression was also increased and the migratory activity was decreased (34). Deprivation of E-cadherin increased migration and invasion and potentiated the metastatic potential of vimentin (35). These results support our findings that the upregulation of E-cadherin suppressed migration and invasion ability.

Some studies indicate that HDAC inhibitors induce EMT (19,22). Our previous study revealed that HDAC inhibitors induced EMT by decreasing E-cadherin through its translocation into the nucleus and potentiated the effect of TGF- $\beta$-induced EMT of colon cancer cells (22). Another study reported that TSA and SAHA induced EMT by upregulating mesenchymal markers (vimentin, N-cadherin, and fibronectin) and stem cell markers [sex determining region Y-box 2 (Sox-2) and Nanog] through the activation of EMT-TFs (Zeb1, Zeb2, and Slug) in prostate cancer cells (19). In contrast, a different study indicated that vorinostat inhibited TGF-b1/Smad signaling and increased the expression of the cadherin-1 gene $(C D H 1)$ in biliary cancer cells (23). The authors suggested that vorinostat is associated with the 
A
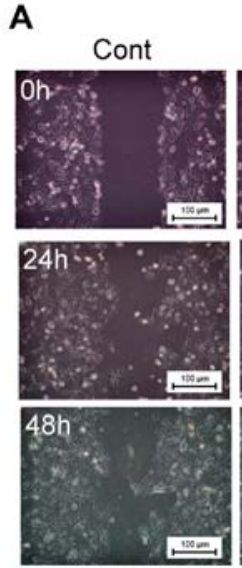

B

Cont

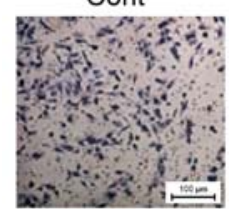

GEM
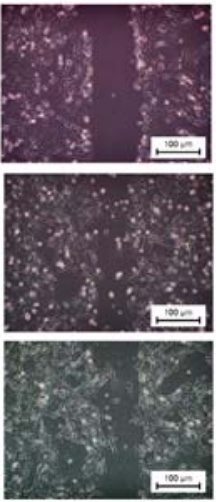

GEM

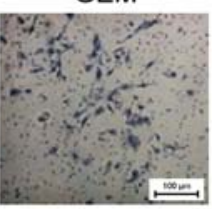

Wound healing assay TSA
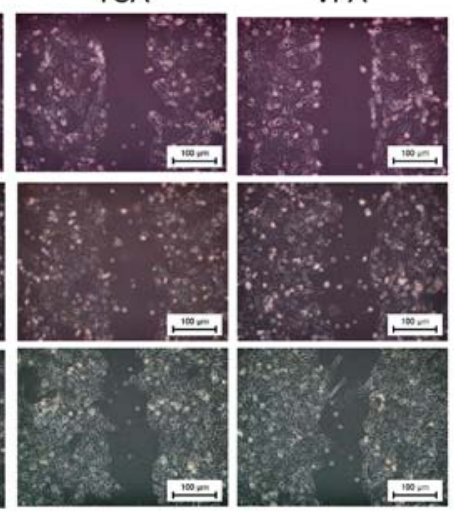

Invasion assay

TSA
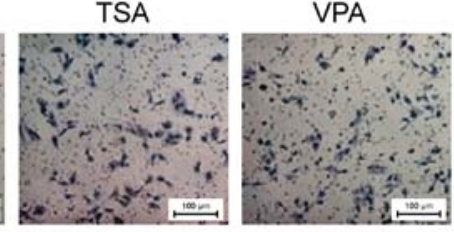

GEM+TSA
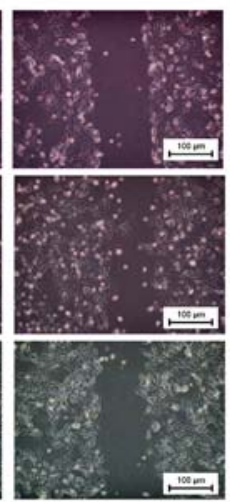

GEM+TSA

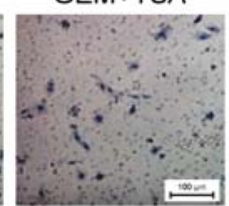

GEM+VPA
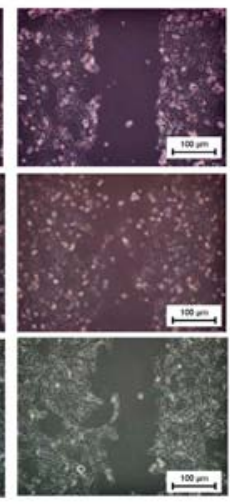

GEM+VPA
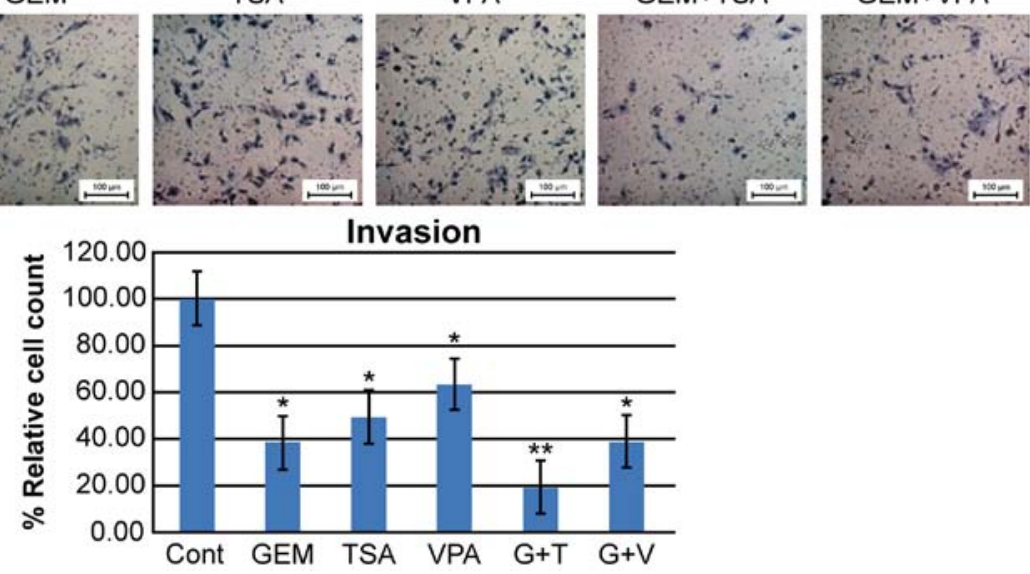

Figure 5. (A) Migration assay of human cholangicellular carcinoma HuCC-T1 cells treated with trichostatin A (TSA, $200 \mathrm{nM}$ ) or valproic acid (VPA, $0.5 \mathrm{mM}$ ) for $48 \mathrm{~h}$. Treatment with TSA alone decreased migration ability whereas VPA did not show any difference compared to the control levels. Migration ability was increased in the cells treated with gemcitabine (GEM) alone, but decreased following co-treatment with TSA or VPA. (B) Invasion assay of HuCC-T1 cells treated with TSA or VPA with or without GEM for $24 \mathrm{~h}$. Invasion of HuCC-T1 cells was decreased by TSA or VPA alone combined with GEM ("P<0.05; $\left.{ }^{* *} \mathrm{P}<0.01\right)$. Data are shown as means \pm standard error (SE).

A

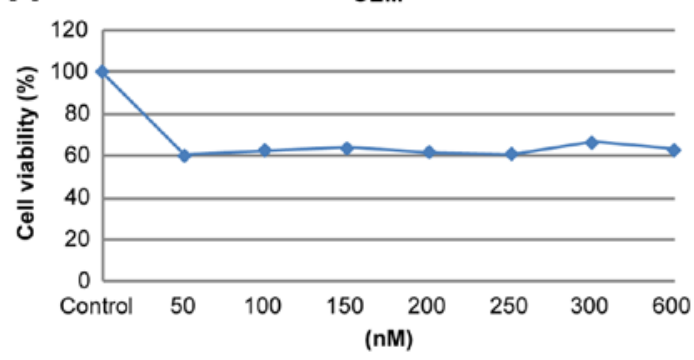

C

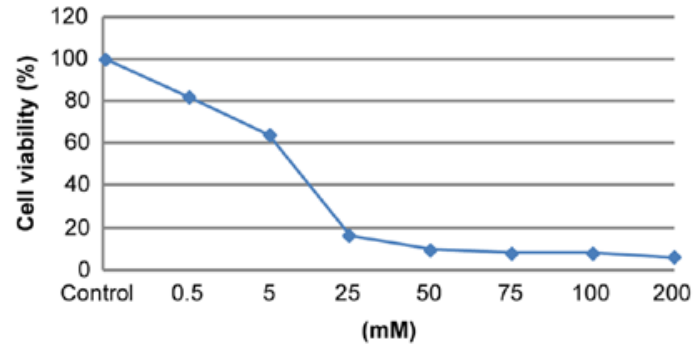

B

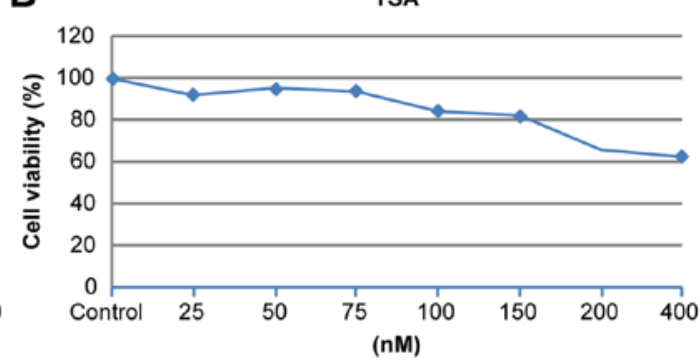

D

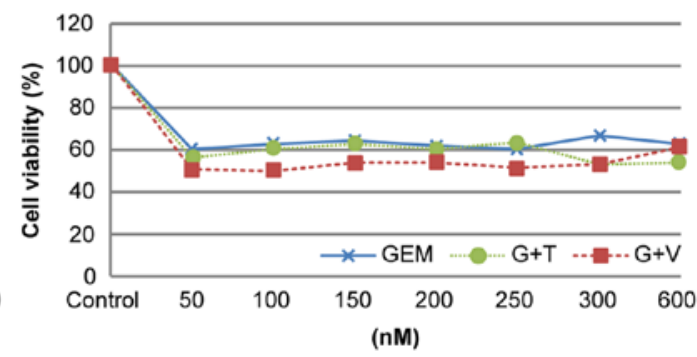

Figure 6. Cell proliferation assay of human cholangiocellular carcinoma HuCC-T1 cells. (A) gemcitabine (GEM), (B) trichostatin A (TSA), (C) valproic acid (VPA) and (D) co-treatment with GEM and TSA $200 \mathrm{nM}$ or VPA $0.5 \mathrm{mM}$. GEM $50 \mathrm{nM}$, TSA $200 \mathrm{nM}$, or VPA $0.5 \mathrm{mM}$ inhibited cell proliferation. However, treatment with GEM combined with TSA or VPA did not affect cell proliferation compared to treatment with GEM alone. G+T, GEM and TSA; $\mathrm{G}+\mathrm{V}, \mathrm{GEM}$ and VPA. 
downregulation of mesenchymal markers such as Snail, Zeb, and Twist, indicating the suppression of EMT (23). These discrepant results may have originated from the differential effect of HDAC inhibitors on the various cell types and the expression pattern of the HDACs, as well as the type of HDAC inhibitor and its dose. Other factors that could be implicated include non-histone proteins that may act as substrates of HDACs including transcription factors, transcription regulators and signal mediators (36). Therefore, the effect of HDAC inhibitors might be associated with multiple pathways that are directly or indirectly regulated by HDACs.

Co-treatment with HDAC inhibitors and conventional chemotherapy has been reported to exert a synergistic effect on cancer cell proliferation (37). Although the combination of HDAC inhibitors and chemotherapeutic agents has been shown to be effective, the results of the co-treatment vary in effectiveness according to the type of HDAC inhibitor, the particular chemotherapeutic agent, the specific tumor type, and the genetic alterations of the tumor. The mechanisms by which HDAC inhibitors potentiate the effect of chemotherapeutics in specific disease are largely unknown. A plausible mechanism is that the HDAC inhibitor-mediated DNA decondensation increases chromatin accessibility and subsequently facilitates access of DNA-damaging agents to their substrate. Alternatively, chemotherapeutic agents may alter EMT-TFs and EMT-related signaling pathways, thereby potentiating the effectiveness of HDAC inhibitors. A recent study reported that co-treatment with vorinostat and GEM improved the survival time in a GEM-resistant xenograft mouse model (23). In this study, TSA or VPA showed limited suppression of GEM-induced EMT in CCA cells.

In conclusion, we suggest that TSA and VPA in combination with GEM suppressed the migration and invasion of CCA cells, with tolerable cytotoxicity. However, these HDAC inhibitors augmented both E-cadherin and vimentin expression, and the effects were variable in CCA cells. Therefore, the clinical application of HDAC inhibitors in the treatment of biliary cancer should be considered with caution.

\section{Acknowledgements}

Not applicable.

\section{Funding}

The present study was supported by a grant from the National R\&D Program for Cancer Control, Ministry of Health and Welfare, Republic of Korea (no. 1120330).

\section{Availability of data and materials}

The datasets used during the present study are available from the corresponding author upon reasonable request.

\section{Authors' contributions}

JHW and SMP designed this study and wrote the manuscript. EJL and MJ performed experiments and interpreted the results. SMP reviewed and edited the manuscript. All authors read and approved the manuscript and agree to be accountable for all aspects of the research in ensuring that the accuracy or integrity of any part of the work are appropriately investigated and resolved.

\section{Ethics approval and consent to participate}

Not applicable.

\section{Consent for publication}

Not applicable.

\section{Competing interests}

The authors declare that they have no competing interests.

\section{References}

1. Hezel AF and Zhu AX: Systemic therapy for biliary tract cancers. Oncologist 13: 415-423, 2008.

2. Limpaiboon T: Epigenetic aberrations in cholangiocarcinoma: Potential biomarkers and promising target for novel therapeutic strategies. Asian Pac J Cancer Prev 13(Suppl): 41-45, 2012.

3. Ropero $S$ and Esteller M: The role of histone deacetylases (HDACs) in human cancer. Mol Oncol 1: 19-25, 2007.

4. Marchion D and Münster P: Development of histone deacetylase inhibitors for cancer treatment. Expert Rev Anticancer Ther 7: 583-598, 2007.

5. Xu LN, Wang X and Zou SQ: Effect of histone deacetylase inhibitor on proliferation of biliary tract cancer cell lines. World J Gastroenterol 14: 2578-2581, 2008.

6. Sriraksa R and Limpaiboon T: Histone deacetylases and their inhibitors as potential therapeutic drugs for cholangiocarcinomacell line findings. Asian Pac J Cancer Prev 14: 2503-2508, 2013.

7. Iwahashi S, Ishibashi H, Utsunomiya T, Morine Y, Ochir TL, Hanaoka J, Mori H, Ikemoto T, Imura S and Shimada M: Effect of histone deacetylase inhibitor in combination with 5-fluorouracil on pancreas cancer and cholangiocarcinoma cell lines. J Med Invest 58: 106-109, 2011.

8. Kalluri R and Weinberg RA: The basics of epithelial-mesenchymal transition. J Clin Invest 119: 1420-1428, 2009.

9. Vleminckx K, Vakaet L Jr, Marreel M, Fiers W and van Roy F: Genetic manipulation of E-cadherin expression by epithelial tumor cells reveals an invasion suppressor role. Cell 66: 107-119, 1991.

10. Di Fazio P, Montalbano R, Quint K, Alinger B, Kemmerling R, Kiesslich T, Ocker M and Neureiter D: The pan-deacetylase inhibitor panobinostat modulates the expression of epithelialmesenchymal markers in hepatocellular carcinoma models. Oncol Lett 5: 127-134, 2013.

11. Shah P, Gau Y and Sabnis G: Histone deacetylase inhibitor entinostat reverses epithelial to mesenchymal transition of breast cancer cells by reversing the repression of E-cadherin. Breast Cancer Res Treat 143: 99-111, 2014.

12. Srivastava RK, Kurzrock R and Shankar S: MS-275 sensitizes TRAIL-resistant breast cancer cells, inhibits angiogenesis and metastasis, and reverses epithelial-mesenchymal transition in vivo. Mol Cancer Ther 9: 3254-3266, 2010.

13. Taylor MD, Liu Y, Nagji AS, Theodosakis N and Jones DR: Combined proteasome and histone deacetylase inhibition attenuates epithelial-mesenchymal transition through E-cadherin in esophageal cancer cells. J Thorac Cardiovasc Surg 139: 1224-1232, 2010

14. Meng F, Sun G, Zhong M, Yu Y and Brewer MA: Anticancer efficacy of cisplatin and trichostatin A or 5-aza-2'-deoxycytidine on ovarian cancer. Br J Cancer 108: 579-586, 2013.

15. Kaimori A, Potter JJ, Choti M, Ding Z, Mezey E and Koteish AA: Histone deacetylase inhibition suppresses the transforming growth factor beta1-induced epithelial-to-mesenchymal transition in hepatocytes. Hepatology 52: 1033-1045, 2010.

16. Xiao W, Chen X, Liu X, Luo L, Ye S and Liu Y: Trichostatin A, a histone deacetylase inhibitor, suppresses proliferation and epithelial-mesenchymal transition in retinal pigment epithelium cells. J Cell Mol Med 18: 646-655, 2014. 
17. Chen X, Xiao W, Chen W, Luo L, Ye S and Liu Y: The epigenetic modifier trichostatin A, a histone deacetylase inhibitor, suppresses proliferation and epithelial-mesenchymal transition of lens epithelial cells. Cell Death Dis 4: e884, 2013.

18. Yoshikawa M, Hishikawa K, Marumo T and Fujita T: Inhibition of histone deacetylase activity suppresses epithelial-tomesenchymal transition induced by TGF-betal in human renal epithelial cells. J Am Soc Nephrol 18: 58-65, 2007.

19. Kong D, Ahmad A, Bao B, Li Y, Banerjee S and Sarkar FH: Histone deacetylase inhibitors induce epithelial to mesenchymal transition in prostate cancer cells. PLoS One 7: e45045, 2012.

20. Giudice FS, Pinto DS Jr, Nör JE, Squarize CH and Castilho RM: Inhibition of histone deacetylase impacts cancer stem cells and induces epithelial-mesenchyme transition of head and neck cancer. PLoS One 8: e58672, 2013.

21. Jiang GM, Wang HS, Zhang F, Zhang KS, Liu ZC, Fang R, Wang H, Cai SH and Du J: Histone deacetylase inhibitor induction of epithelial-mesenchymal transitions via up-regulation of Snail facilitates cancer progression. Biochim Biophys Acta 1833: 663-671, 2013

22. Ji M, Lee EJ, Kim KB, Kim Y, Sung R, Lee SJ, Kim DS and Park SM: HDAC inhibitors induce epithelial-mesenchymal transition in colon carcinoma cells. Oncol Rep 33: 2299-2308, 2015.

23. Sakamoto T, Kobayashi S, Yamada D, Nagano H, Tomokuni A, Tomimaru Y, Noda T, Gotoh K, Asaoka T, Wada H, et al: A histone deacetylase inhibitor suppresses epithelial-mesenchymal transition and attenuates chemoresistance in biliary tract cancer. PLoS One 11: e0145985, 2016.

24. Yao X, Wang X, Dai L, Wang Z, Zhang G, Yan Q and Zhou W: Clinicopathological and prognostic significance of epithelial-mesenchymal transition related-protein in intrahepatic cholangiocarcinoma. Onco Targets Ther 5: 255-261, 2012.

25. Vaquero J, Guedj N, Clapéron A, Nguyen Ho-Bouldoires TH, Paradis V, Fouassier L: Epithelial-mesenchymal transition in cholangiocarcinoma: From clinical evidence to regulatory networks. J Hepatol 66: 424-441, 2017.

26. Ku JL, Yoon KA, Kim IJ, Kim WH, Jang JY, Suh KS, Kim SW, Park YH, Hwang JH, Yoon YB and Park JG: Establishment and characterisation of six human biliary tract cancer cell lines. Br J Cancer 87: 187-193, 2002.

27. Sipos F and Galamb O: Epithelial-to-mesenchymal and mesenchymal-to-epithelial transitions in the colon. World J Gastroenterol 18: 601-608, 2012.
28. Rittling SR and Baserga R: Functional analysis and growth factor regulation of the human vimentin promoter. Mol Cell Biol 7: 3908-3915, 1987.

29. Lilienbaum A and Paulin D: Activation of the human vimentin gene by the Tax human T-cell leukemia virus I: Mechanisms of regulation by the NF-kappa B transcription factor. J Biol Chem 268: 2180-2188, 1993.

30. Chen JH, Vercamer C, Li Z, Paulin D, Vandenbunder B and Stehelin D: PEA3 transactivates vimentin promoter in mammary epithelial and tumor cells. Oncogene 13: 1667-1675, 1996.

31. Salvetti A, Lilienbaum A, Li Z, Paulin D and Gazzolo L: Identification of a negative element in the human vimentin promoter: Modulation by the human T-cell leukemia virus type I Tax protein. Mol Cell Biol 13: 89-97, 1993.

32. Wu Y, Zhang $X$, Salmon $M$ and Zehner ZE: The Zinc finger repressor, ZBP-89, recruits histone deacetylase 1 to repress vimentin gene expression. Gene Cells 12: 905-918, 2007.

33. Wang X, Xu J, Wag H, Wu L, Yuan W, Du J and Cai S: Trichostatin A, a histone deacetylase inhibitor, reverses epithelialmesenchymal transition in colorectal cancer SW480 and prostate cancer PC3 cells. Biochem Biophys Res Commun 456: 320-326, 2015.

34. Han RF, Li K, Yang ZS, Chen ZG and Yang WC: Trichostatin A induces mesenchymal-like morphological change and gene expression but inhibits migration and colony formation in human cancer cells. Mol Med Rep 10: 3211-3216, 2014.

35. Techasen A, Loilome W, Namwat N, Khuntikeo N, Puapairoj A, Jearanaikoon P, Saya $\mathrm{H}$ and Yongvanit P: Loss of E-cadherin promotes migration and invasion of cholangiocarcinoma cells and serves as a potential marker of metastasis. Tumor Biol 35: 8645-8652, 2014

36. Xu W, Parmigiani R and Marks P: Histone deacetylase inhibitors: Molecular mechanisms of action. Oncogene 26: 5541-5552, 2007.

37. Donadelli M, Costanzo C, Beghelli S, Scupoli MT, Dandrea M, Bonora A, Piacentini P, Budillon A, Caraglia M, Scarpa A and Palmieri M: Synergistic inhibition of pancreatic adenocarcinoma cell growth by trichostatin A and gemcitabine. Biochim Biophys Acta 1773: 1095-1106, 2007. 